\title{
Una aproximación a la medición de la percepción de prácticas de crianza para adolescentes
}

\section{An approach to measuring the perception of teen parenting practices}

Mirta Margarita Flores Galaz

María de Lourdes Cortés Ayala

Universidad Autónoma de Yucatán

José González Tovar

Universidad de Coahuila

Melissa García Meraz

Universidad Autónoma del Estado

de Hidalgo
Johannes Oudhof Van Barneveld

Universidad Autónoma del Estado

de México

Martha Frías Armenta

Universidad de Sonora

Georgina Lozano Lazo

Javier Zavala Rayas

Universidad Autónoma de Zacatecas

\section{Resumen}

El presente estudio tuvo como objetivo construir y validar una escala para medir la percepción de la crianza paterna en adolescentes mexicanos. Participaron en él 432 adolescentes, seleccionados mediante un muestreo no probabilístico; ellos provenían de seis estados de la República Mexicana (180 de Sonora, Toluca e Hidalgo, 60 eran cada uno; 100 de Zacatecas; 103 de Yucatán; y 49 de Coahuila). Del total de la muestra, 187 eran mujeres y 185 eran hombres (60 personas no dieron esta información). La edad promedio fue $14.15(D E=1.28)$. La Escala de Percepción de Prácticas de Crianza para Adolescentes, elaborada y administrada, consta de 63 afirmaciones tipo Likert con cinco opciones de respuesta $(1=$ Nunca a $5=$ Siempre), que evalúan la frecuencia (de Siempre a Nunca) de diversas conductas que los padres y madres realizan cotidianamente para educar a sus hijos. Los resultados obtenidos a través del análisis factorial exploratorio mostraron que para la subescala de Mamá se conformaron cuatro factores: Cuidado y calidez $(\mathrm{alfa}=0.93)$, Transmisión de valores $($ alfa $=$ 0.83), Apoyo y orientación escolar (alfa $=0.73$ ), así como Control conductual (alfa $=0.57)$; mientras que para la subescala de Papá se encontraron siete factores, aunque cinco presentan congruencia conceptual: Cuidado y calidez (alfa 0.95), Transmisión de valores (alfa $=0.73$ ), Disciplina inductiva $($ alfa $=0.70)$, Apoyo y orientación escolar $($ alfa $=0.72)$, y además de Control conductual (alfa $=$ 0.57). Asimismo, fueron analizadas las propiedades psicométricas del instrumento y se presentaron las diferencias encontradas por las variables criterio (sexo, zona geográfica).

Palabras clave: crianza, padres, adolescentes, familia, medición.

Nota del autor

Mirta Margarita Flores Galaz, Facultad de Psicología, Universidad Autónoma de Yucatán (UADY); María de Lourdes Cortés Ayala, Facultad de Psicología, UADY; José González Tovar, Facultad de Psicología, Universidad de Coahuila; Melissa García Meraz, Departamento de Psicología, Universidad Autónoma del Estado de Hidalgo; Johannes Oudhof Van Barneveld, Facultad de Ciencias de la Conducta, Universidad Autónoma del Estado de México; Martha Frías Armenta, Departamento de Derecho, Universidad de Sonora; Georgina Lozano Lazo, Facultad de Psicología, Universidad Autónoma de Zacatecas (UAZ); Javier Zavala Rayas, Facultad de Psicología, UAZ.

La correspondencia en relación con este artículo debe dirigirse a Mirta Margarita Flores Galaz, Facultad de Psicología, UADY, km 1 Mérida-Tizimín, S/N, C.P. 97305, Cholul, Yucatán, México.

Dirección electrónica: fgalaz@correo.uady.mz 


\begin{abstract}
This study aimed to construct and validate a scale to measure the perception of parenting for Mexican adolescents. A total of 432 teenagers were selected through a no probabilistic sampling from six states in Mexico: 180 (60 each one) from Sonora, Toluca and Hidalgo; 100 from Zacatecas, 103 from Yucatan and 49 of Coahuila. 187 were women and 185 men (60 persons did not indicate their sex). The average age was 14.15 years with a standard deviation of 1.28. The Perceived of Parenting Practices for Adolescents consists of 63 claims in a 5-point Likert-style scale $(1=$ Never, to $5=$ Always $)$ that assess the perceived frequency of activities than father and mother do every day to educate them according to the adolescents. Results, obtained through the exploratory factor analysis, showed that for the Mom subscale four factors were formed: Care and warmth (alpha $=0.93)$, Transmission of values (alpha $=0.83)$, Support and school guidance $($ alpha $=0.73)$ and Behavioral control (alpha =0.57). The Dad subscale was form by seven factors although only five showed conceptual consistency: Care and warmth (alpha 0.95), Transmission of values (alpha =0.73), Inductive discipline (alpha=0.70), School support and guidance $($ alpha $=0.72)$, Behavioral control $(\mathrm{alpha}=0.57)$. Psychometric properties of the instrument were analized and differences between criterion variables (sex and geographic zone).
\end{abstract}

Keywords: parenting, parents, teenegers, family, assessment.

Los numerosos estudios sobre la familia concuerdan en considerar que, para la mayoría de los individuos, la familia juega un papel fundamental en el proceso de desarrollo ya que, desde el nacimiento hasta la adolescencia, los padres, intencionalmente o no, son la influencia principal en la vida de sus hijos (Flores, Cortés, \& Góngora, 2008). A través de la crianza, los padres (o cuidadores principales de un niño) se involucran en una serie de actividades para contribuir al pleno desarrollo físico y psicosocial de los menores a su cuidado (Bornstein \& Sawyer, 2005). La crianza paterna hace referencia a los conocimientos, actitudes y creencias que los padres asumen en relación con la salud, la nutrición, la importancia de los ambientes físico y social y las oportunidades de aprendizaje de sus hijos en el hogar (Eraso, Bravo, \& Delgado, 2006).

Según Aguirre (2000), "las prácticas de crianza... son un proceso... un conjunto de acciones concatenadas, que cuenta un inicio, que se va desenvolviendo conforme pasa el tiempo" (p. 5). A su vez, Palacios y Moreno (1999) definen la crianza paterna como una tarea compleja y multidimensional, ya que “... los padres tienen que prestar atención a una gran variedad de circunstancias, y las demandas que plantean los hijos son cambiantes" (p. 3). Los estilos de crianza o estilos parentales hacen referencia al conjunto de actitudes y conductas de los padres, quienes crean un clima emocional determinado (Baumrind, 1971; Maccoby \& Martin, 1983), en tanto que las prácticas de 
crianza aluden a las conductas particulares que los padres y madres realizan cotidianamente para conseguir ciertos objetivos educativos con sus hijos (Darling \& Steinberg, 1993).

Los padres de familia usan las estrategias de crianza que creen les permitirán obtener los objetivos educativos deseados, según la situación, las características del niño, la conducta del momento y la cultura; visto así, en el proceso de crianza, los padres imponen límites, inculcan valores y autocontrol, y también proveen calidez (Craig, 2008). Los numerosos estudios en este campo han mostrado dos aspectos o dimensiones que parecen esenciales en la crianza: el control y la calidez o afecto; el control de los padres hace referencia a las restricciones impuestas al niño (p. e., exigir obediencia de ciertas reglas, que cumplan sus responsabilidades, limitar su libertad). La otra dimensión importante es la calidez, referida al grado de afecto y aprobación que exteriorizan; los padres afectuosos con frecuencia sonríen a sus hijos, los abrazan, elogian y alientan. Los padres poco afectuosos u hostiles, critican, ignoran y castigan mucho a sus hijos (Becker, 1964; Maccoby, 1984).

A partir de las dimensiones anteriores, Baumrind $(1975,1980)$ desarrolló una tipología para clasificar los estilos de crianza, y propuso tres estilos o patrones: estilo autoritativo (con autoridad), autoritario y permisivo. Maccoby y Martin (1983) agregan el estilo indiferente. Numerosos estudios se han encargado de evidenciar los beneficios de la crianza basada en el afecto y el control moderado, directivo, de los hijos, característico del estilo denominado autoritativo (Becker, 1964; Block, 1981; Ceballos \& Rodrigo, 1998; Goodnow \& Collins, 1990; Maccoby \& Martin, 1983; Musitu \& Soledad-Lila, 1993; Palacios, González, \& Moreno, 1992).

Asimismo, se ha demostrado que las prácticas de crianza de los padres tienen importantes implicaciones en el desarrollo de los hijos; la investigación al respecto ha mostrado que prácticas positivas centradas en el afecto, comunicación y el apoyo, así como en la promoción de la autonomía (Mestre et al., 1999; Oliva, Parra, \& Arranz, 2008; Ramírez, 2009) se relacionan con: la competencia académica (Balzano, 2002; Peralbo \& Fernández, 2003), el aprendizaje de valores positivos (Grusec \& Goodnow, 1994; Molpereces, Llenares, \& Musitu, 2001), una buena autoestima (Alonso \& Román, 2005; Maccoby \& Martin, 1983), y el desarrollo de la competencia social (Cava, Musitu, \& Murgui, 2006), entre otros.

El estudio de las prácticas de crianza parentales y su impacto en el desarrollo de los hijos ha sido la perspectiva dominante en un incio en este campo de estudio. Más recientemente, el interés se ha dirigido a una dimensión complementaria: las prácticas de crianza desde la perspectiva de los hijos, donde numerosos estudios subrayan su importancia (Carrasco, Holgado, \& Del Barrio, 2007; Grusec \& Goodnow, 1994; Ivanova \& Israel, 2006; Rohner \& Veneziano, 2001). Los 
estudios pioneros de los años 70 de Perron y Mathon (1976), sobre la percepción de las figuras parentales de niños de 3 a 7 años, mostraron que los niños distinguen a la madre como fuente del amor y al padre como una figura de autoridad a partir de los cinco años. Otros estudios mostraron la importancia de la percepción subjetiva de los niños sobre la relación padre-hijo en el funcionamiento interpersonal, por ejemplo, los niños con problemas de comportamiento perciben a sus padres como poco involucrados, punitivos y con comportamiento de crianza negativos (Bronfenbrenner, 1979; Michaels, Meese, \& Stollack, 1983).

Forehand y Nousiainen (1993) reportan, en muestras clínicas, que en los hijos varones, a mayor percepción de conductas maternas de menor aceptación y más afecto negativo, presentan mayor externalización de problemas emocionales; las hijas parecen mostrar una mayor internalización de problemas emocionales/conductuales en relación a una mayor percepción de ansiedad y depresión paterna y niveles más bajos de control maternal. Además, ambos hijos mostraron evidencia de mayores problemas emocionales conductuales en relación con la percepción de triangulación y de conflicto entre los padres. Asimismo, los autores encontraron, en muestras no clínicas, que la percepción de aceptación de los padres y de estilos de crianza positivos contribuyen a un mejor funcionamiento de los adolescentes. En general, la percepción del afecto y de aceptación de los padres en los adolescentes parecen estar fuertemente relacionadas con su funcionamiento y ajuste psicológico (Bosco, Renk, Dinger, Epstein, \& Phares, 2003).

Más recientemente, se ha evidenciado que aun cuando el proceso estudiado se refiere a la percepción del hijo más que a la veracidad de la información (Finley \& Schwartz, 2004), esta perspectiva ha mostrado ser más confiable (Carrasco, Holgado, Del Barrio, \& Barbero, 2007; Samper, Cortés, Mestre, Nácher, \& Tur, 2006) y coherente que la información proporcionada por los padres (González, Cause, \& Mason, 1996; Morris, Silk, Steinberg, Sessa, Avenevoli, \& Essex, 2002) para predecir el ajuste emocional, rendimiento escolar y sintomatología depresiva (Paulson, 1994; Roa \& Del Barrio, 2002; Schaefer, 1965). La percepción de los hijos acerca de las prácticas de crianza de los padres, incluso poseen mayor validez predictiva para la aceptación en el grupo de pares (Gaylord, Kitzmann, \& Coleman, 2003), que otras fuentes. Resultados similares han encontrado Méndez, Andrade y Peñaloza (2013) en estudios con adolescentes mexicanos.

En cuanto al sexo del progenitor, los estudios internacionales coinciden en mostrar diferencias en cuanto a la percepción que se tiene del padre y de la madre (Pipp, Shaver, Jennings, Lamborn, \& Fischer, 1985; Rodrigo, García, Márquez, \& Triana, 2005); las madres son percibidas con más frecuencia como más amorosas, comprensivas y cercanas, aunque más controladoras (Rodríguez, 
Del Barrio, \& Carrasco, 2009; Paulson, Hill, \& Holmbeck, 1991; Paulson \& Sputa, 1996), en tanto que a los padres se les percibe más autoritarios, rígidos y severos (Paulson \& Sputa, 1996; Shek, 1998, 2000; Sorbring, RödholmFunnemark, \& Palmerus, 2003). En cuanto al sexo de los hijos, se ha encontrado que los varones perciben a sus padres más estrictos y las mujeres perciben a sus madres más afectuosas y menos estrictas (Sorbring et al., 2003). Por edad, las investigaciones señalan que a medida que los hijos son mayores perciben menos apoyo e implicación en ambos padres (Spera, 2005; Steinberg, 2001). En sí, la crianza paterna se percibe diferencialmente mediada por el sexo y la edad (Rodríguez, Del Barrio, \& Carrasco, 2009).

Otros estudios evidencian que si los padres son percibidos por sus hijos adolescentes como más autoritarios y violentos al comunicarse, y se percibe más inflexibilidad familiar, hay un mayor aumento de conflicto entre padres-hijos (Pérez \& Aguilar, 2009). Por el contrario, la percepción de una paternidad autoritativa se relaciona con contextos familiares con bajo conflicto, y menor número de problemas en la adolescencia temprana (McKinney, Milone, \& Renk, 2011; Pérez \& Alvarado, 2015).

En México, los estudios con niños de primaria realizados en Yucatán (Flores, Cortés, \& Góngora, 2008), encuentran que las niñas perciben a su papá más afectuoso que los niños, en tanto éstos perciben que imparte más disciplina punitiva y es más intolerante, además de ejercer más control. En cuanto a la mamá, las niñas perciben que impone más disciplina, les apoya menos en la escuela, les da más afecto y es más tolerante con ellas que con los niños. Estudios realizados en Toluca (Rodríguez, Oudhof van Barneveld, González-Arratia, \& Unikel, 2011), encontraron datos semejantes.

Otros estudios con población mexicana se han interesado en la percepción del apoyo recibido de los padres (Aguilar, Valencia, \& Romero, 2004; Palacios \& Andrade, 2006; Rivera \& Andrade, 1998, 2010; Vallejo, 2002). Por su parte, Villegas-Pantoja, Alonso-Castillo, Alonso-Castillo, y Martínez-Maldonado (2014), en un estudio con adolescentes mexicanos, concluyeron que una mayor percepción de conductas de calidez en la madre se relaciona con una más tardía edad en el inicio o consumo de sustancias. Los estudios sobre la percepción del control parental de Betancourt y Andrade-Palos (2011), muestran que el control psicológico de ambos padres tiene una mayor influencia que el control conductual en la presencia de problemas emocionales y de conducta, aunque se obtienen diferencias entre hombres y mujeres.

La revisión de la literatura internacional evidenciada en el Handbook of Family Measurement Techniques muestra que existen numerosos instrumentos desarrollados desde 1975 a la fecha para la medición de diversos aspectos de la conducta parental (Touliatos, Permutter, \& Straus, 2001). En México, se han diseñado pocos instrumentos con base en la percepción de la crianza paterna, entre éstos 
destacan la Escala para las Prácticas Parentales de Crianza Materna (Jiménez, HernandezGuzmán, \& Reidl 2001), dirigida a niños preescolares; la escala para la Evaluación de las Relaciones Familiares, también dirigida a los hijos (Rivera \& Andrade, 1998); y el Inventario de Stress en la crianza, que constan de dos dimensiones: la dirigida al niño y la dirigida a los padres (Montiel, Vera, Peña, Rodríguez, \& Félix, 2002). Recientemente, Aguilar (2003) ha elaborado una escala se estilos parentales para estudiantes de nivel medio y superior, basada en los trabajos de Baumrind (1991) y de Buri (1991). El estilo de crianza y la paternidad punitiva también han sido explorados $\mathrm{y}$ asociados a la depresión en madres mexicanas (Frías, Sotomayor, Corral, \& Castell, 2004). Por su parte, Oudhof, González, Rodríguez y Unikel (2011) han desarrollado la Escala de Percepción de Crianza dirigida a jóvenes, conformada por tres factores: interés en las actividades del hijo, apoyo y orientación. A pesar de contar con estas escalas, ninguna de ellas ha sido construida y validada, según población de diferentes regiones geográficas del país. Dada la importancia de la percepción de la crianza paterna en el desarrollo de los individuos, el objetivo de este trabajo fue construir y validar una escala para medir la percepción de la crianza para adolescentes mexicanos de diversos estados del país, y de igual forma, establecer las diferencias entre la percepción de Papá y Mamá y las diferencias por sexo y zona geográfica.

\section{Método}

\section{Participantes}

Mediante un muestreo no probabilístico de seis estados de la República Mexicana fueron seleccionados 432 adolescentes: 60 de Sonora, Toluca e Hidalgo, 100 de Zacatecas y 103 de Yucatán. De éstos 187 eran mujeres y 185, hombres (60 personas no señalaron su sexo). La edad promedio fue 14.15 años con una desviación estándar de 1.28.

\section{Instrumento}

Escala de Percepción de Prácticas de Crianza para Adolescentes. Consta de 63 afirmaciones tipo Likert de cinco opciones de respuesta, éstas evalúan la frecuencia de Siempre a Nunca con la que los hijos perciben lo que el padre y madre realizan día a día en cuanto a una serie de acciones para educarlos. Es importante señalar que la escala se construyó a partir de un estudio exploratorio realizado en diferentes zonas geográficas del país. El objetivo fue explorar la percepción de la crianza paterna (papá y mamá), a través de un cuestionario de preguntas abiertas. Con base en el análisis de contenido realizado, y de la revisión teórica fueron conformados siete factores con sus respectivos reactivos: 1 . Apoyo, afecto y motivación (11 reactivos), 2. Límites y reglas (12 reactivos), 3. Castigo verbal (7 reactivos), 4. Castigo físico (9 reactivos), 5. Comunicación (7 reactivos), 6. Apoyo escolar (6 reactivos), 7. Orientación y formación (11 reactivos). A continuación se describen: 
1. Apoyo, afecto y motivación. Consiste en tener una implicación afectiva con el hijo y atender sus necesidades y deseos. Los reactivos que lo conforman son: Me muestra cariño (abraza, besa, apapacha), me brinda confianza, me muestra comprensión, me hace saber que soy importante para la familia, me ayuda a resolver los problemas personales que tengo, me hace sentir que puedo contar con él (ella), me dice que me quiere, me apoya cuando lo necesito, me da palabras de aliento, me dice siempre lo orgulloso que está de mí, me anima a seguir adelante a pesar de todo.

2. Límites y reglas. Implica el establecimiento claro de pautas de conducta deseables. Los reactivos que incluye son: Me corrige cuando cometo un error, me explica las reglas que hay en la casa, me llama la atención cuando desobedezco, me premia cuando hago algo bueno, me quita privilegios o premios (por ejemplo, ipod, televisión, celular), me prohíbe ir a casa de mis amigos (as), me prohíbe hacer ciertas cosas que están mal, me pide que le diga a dónde voy cuando salgo, me pide que le diga con quién paso mi tiempo libre, me dice que le diga a qué hora regreso en la noche, y la última, me dice que puedo tener amigos en la casa cuando no haya adultos en ella.

3. Castigo verbal. Son las pautas verbales aversivas para disminuir la frecuencia de conductas indeseables. Los reactivos son: me grita; me amenaza con pegarme aunque no lo haga; me dice groserías, malas palabras o maldiciones; me llama tonto (a), flojo (a) o algo parecido; me dice que me correrá de la casa; me dice que me correrá de la casa a golpes (patadas); y finalmente, me ignora, no me habla.

4. Castigo físico. Son las pautas instrumentales aversivas para disminuir la frecuencia de conductas indeseables. Incluye: me golpea con la mano; me pega en la mano, brazo o pierna; me pega con el cinturón, peine o palo; me pega en la cara (cachetadas), cabeza o me jala de las orejas; me pega en el estómago, pecho o espalda; me da patadas; me jala o avienta; me pega constantemente y durante algunos minutos; así como me agarra con fuerza del cuello o de la nuca.

5. Comunicación. Implica establecer una comunicación permanente y bidireccional entre padres e hijos. Los reactivos que la incluyen son: trata de entender lo que me sucede; toma tiempo para platicar conmigo; vemos la televisión juntos y platicamos sobre los programas; me escucha cuando necesito contarle algo; me pregunta qué me sucede cuando estoy triste; está enterado cuando tengo problemas; además de que me dice que le pregunte si tengo dudas de algo.

6. Apoyo escolar. Conlleva el fomentar $y$ favorecer las actividades escolares del hijo y estar al pendiente de su educación. Los reactivos son: se asegura que vaya diariamente a la escuela; se pone a estudiar conmigo; platica con los maestros sobre 
cómo voy en la escuela; me ayuda a hacer la tarea; me compra los útiles que necesito; y me hace ver la importancia de la escuela.

7. Orientación y formación. Proporcionar enseñanzas y consejos para la inserción adecuada en la vida social. Incluye: me inculca valores; me enseña a respetar a los demás; me hace ver cuáles son mis responsabilidades; me pone el ejemplo con su propia conducta; me hace notar mis errores en la convivencia con los demás; me hace ver las consecuencias de mis actos; me enseña a cuidar mi salud; platica conmigo sobre las normas y principios de la vida; me explica por qué algo es incorrecto; me dice lo que está bien o lo que está mal; y me enseña a esforzarme.

\section{Procedimiento}

La aplicación del instrumento se realizó en diferentes escuelas secundarias y preparatorias públicas, seleccionadas por conveniencia de las diferentes sedes participantes, previa autorización. Los adolescentes que participaron eran quienes se encontraban al momento de la aplicación en los salones de clase; se incluyeron adolescentes que vivieran con ambos padres o solo con alguno de ellos. Se les explicó el objetivo del estudio, se les pidió responder con veracidad y se les garantizó el anonimato de sus respuestas. El tiempo promedio de respuesta fue de 20 minutos.

\section{Análisis de datos}

En primer lugar, se realizó un análisis de la distribución de frecuencias de cada uno de los reactivos, así como también el análisis del sesgo, curtosis y discriminación de reactivos por medio de la prueba $t$ de student, además del análisis de consistencia interna por medio del alfa de Cronbach. Enseguida se procedió a obtener un análisis factorial exploratorio de componentes principales con rotación ortogonal, con el objetivo de reducir el número de variables y evidenciar validez de constructo (Kerlinger \& Lee, 2002). En este caso se realizó un análisis para la subescala de Mamá y otro para la subescala de Papá, y las consistencias internas para cada uno de los factores encontrados, además de las medias de tendencia central y dispersión para cada factor. Para conocer las diferencias por sexo se obtuvo una prueba $t$ de student; por zona geográfica un análisis de varianza de una sola vía.

\section{Resultados}

Se consideraron aquellos reactivos que cumplieran con el criterio de tener un sesgo y curtosis ( $\leq$ a 1$)$, de igual manera, se consideró que los reactivos discriminaban siempre y cuando la probabilidad obtenida en la prueba $t$ de Student fuera $\leq 0.05$. Los resultados obtenidos para la subescala de Mamá mostraron que solo 18 reactivos cumplían con el criterio de simetría y 22 con la curtosis. Para la subescala de Papá, 27 cumplieron con el criterio de simetría y 35 con 
el de la curtosis. Es importante mencionar que para la subescala de mamá no discriminaron 18 reactivos, y en la subescala de Papá, 17, es decir, coincidieron en 17 de éstos, de tal manera que no se consideraron para análisis posteriores.

Para la subescala de Mamá se obtuvo un Índice de adecuación del muestreo KaiserMeyer y Olkin KMO de 0.931 y un test de esfericidad de Bartlett de 6388.450; $p=.000$.
Se encontró que los siete factores explicaban $53.56 \%$ de la varianza total acumulada después de la rotación. Sin embargo, al analizar la matriz de estructura factorial y elegir aquellos factores con reactivos, cuyo peso factorial fuera de más menos 0.40 , únicamente se observaron cuatro factores que estaban conformados al menos por tres reactivos y eran congruentes teóricamente (ver tabla 1).

\section{Tabla 1}

Matriz de estructura factorial de la Escala de Percepción de Prácticas de Crianza para Adolescentes para la subescala de Mamá

\begin{tabular}{|c|c|c|c|c|c|c|c|}
\hline \multirow{2}{*}{ Reactivos } & \multicolumn{7}{|c|}{ Factores } \\
\hline & 1 & 2 & 3 & 4 & 5 & 6 & 7 \\
\hline 62. Me hace saber que soy importante para la familia & .710 & .170 & .218 & .112 & .058 & .314 & -.158 \\
\hline 34. Me brinda confianza & .707 & .268 & .147 & .055 & -.022 & -.066 & .156 \\
\hline 18. Me dice que me quiere & .696 & .086 & .208 & .016 & .071 & .117 & -.114 \\
\hline 53. Me muestra comprensión & .687 & .302 & .141 & -.115 & .023 & -.022 & .159 \\
\hline 55. Me muestra cariño (abraza, besa, apapacha) & .683 & -.001 & .170 & -.076 & .239 & .303 & -.096 \\
\hline 43.Me dice lo orgullosa que está de mí & .681 & .106 & .262 & .055 & -.146 & .147 & -.186 \\
\hline 45. Trata de entender lo que me sucede & .661 & .177 & .225 & .232 & -.029 & .106 & -.037 \\
\hline 40. Me pregunta qué me sucede cuando estoy triste & .660 & .124 & .318 & .106 & .068 & .006 & .179 \\
\hline 6. Me escucha cuando necesito contarle algo & .637 & .174 & .153 & .194 & -.054 & -.142 & .090 \\
\hline 10. Me anima a seguir adelante a pesar de todo & .620 & .177 & .039 & .136 & .055 & .013 & .154 \\
\hline 23. Me hace sentir que puedo contar con ella & .580 & .327 & .020 & .307 & .055 & .103 & -.010 \\
\hline 54. Me enseña a esforzarme & .551 & .303 & .303 & .099 & .167 & .098 & .207 \\
\hline 20. Me ayuda a resolver los problemas personales que tengo & .547 & .207 & .357 & .239 & .068 & -.208 & .160 \\
\hline 59. Me da palabras de aliento & .538 & .157 & .386 & -.024 & .189 & .073 & .169 \\
\hline 36. Me dice que le pregunte si tengo dudas de algo & .524 & .314 & .350 & .054 & .026 & -.011 & .229 \\
\hline 3. Me ignora, no me habla & -.521 & .031 & -.036 & -.181 & .394 & .023 & .292 \\
\hline 51. Me apoya cuando lo necesito & .498 & .399 & .217 & .047 & .112 & .061 & .236 \\
\hline 60. Me enseña a cuidar mi salud & .496 & .313 & .021 & .102 & .238 & .360 & .067 \\
\hline 42. Está enterada cuando tengo problemas & .472 & .196 & .408 & .132 & .087 & -.075 & -.048 \\
\hline 2. Me hace ver mis responsabilidades & .158 & .666 & .048 & .090 & .004 & -.038 & -.260 \\
\hline 38. Me compra los útiles que necesito & .198 & .665 & .080 & .065 & -.220 & .336 & .138 \\
\hline 41. Me hace ver la importancia de la escuela & .276 & .636 & .110 & .114 & -.053 & .347 & -.089 \\
\hline 37. Me llama la atención cuando desobedezco & .041 & .635 & .071 & -.008 & .232 & .038 & .040 \\
\hline 35. Me inculca valores & .456 & .572 & .210 & .169 & .037 & .100 & .065 \\
\hline
\end{tabular}


Continuación tabla 1

Reactivos
21. Me dice lo que está bien o lo que está mal
32. Me corrige cuando cometo un error
22. Me explica las reglas que hay en la casa
12. Me hace ver las consecuencias de mis actos
46. Se pone a estudiar conmigo
15. Me ayuda a hacer la tarea
63. Platica con los maestros sobre cómo voy en la escuela
49. Toma tiempo para platicar conmigo
33. Vemos la televisión juntos y platicamos sobre los programas
29. Me dice que le diga a qué hora regreso en la noche
13. Me pide que le diga con quién paso mi tiempo libre
44. Me pide que le diga a dónde voy cuando salgo
27. Me pone el ejemplo con su propia conducta
25. Me hace notar mis errores en la convivencia con los demás
57. Me prohíbe hacer ciertas cosas que están mal
48. Se asegura que vaya diariamente a la escuela
8. Me dice que puedo tener amigos en la casa, aun cuando no hay
adultos en ella
Porcentaje de Varianza Explicada
Alfa de Cronbach
Media
Desviación estándar
Número de reactivos

Número de reactivos

\begin{tabular}{|c|c|c|c|c|c|c|}
\hline \multicolumn{7}{|c|}{ Factores } \\
\hline 1 & 2 & 3 & 4 & 5 & 6 & 7 \\
\hline .296 & .559 & .052 & .240 & .172 & .106 & .046 \\
\hline .384 & .517 & .057 & .156 & .226 & -.012 & -.072 \\
\hline .160 & .428 & .366 & .132 & .194 & -.019 & -.033 \\
\hline .220 & .401 & .173 & .214 & .343 & -.236 & -.051 \\
\hline .238 & .066 & .763 & .009 & -.171 & -.019 & .004 \\
\hline .150 & .110 & .727 & .081 & -.073 & .008 & .024 \\
\hline .161 & .028 & .633 & .105 & .170 & .259 & -.290 \\
\hline .432 & .158 & .469 & .246 & -.042 & .019 & .237 \\
\hline .297 & .121 & .443 & .105 & .188 & .065 & .096 \\
\hline .017 & .192 & .039 & .743 & .053 & .146 & .051 \\
\hline .144 & -.008 & .131 & .629 & .184 & -.109 & -.011 \\
\hline .097 & .084 & .026 & .614 & .091 & .272 & .230 \\
\hline .122 & .222 & .112 & .435 & -.121 & -.178 & -.126 \\
\hline .074 & .171 & .034 & .043 & .624 & -.114 & -.114 \\
\hline .007 & .076 & -.001 & .251 & .589 & .333 & -.016 \\
\hline .190 & .319 & .150 & .073 & -.008 & .668 & .011 \\
\hline .143 & -.133 & .064 & .107 & -.154 & .000 & .588 \\
\hline 19.22 & 10.04 & 8.21 & 5.47 & 4.13 & 3.50 & 2.98 \\
\hline 0.93 & 0.83 & 0.73 & 0.57 & & & \\
\hline 4.22 & 4.46 & 3.27 & 3.72 & & & \\
\hline 0.76 & 0.57 & 0.89 & 0.83 & & & \\
\hline 18 & 9 & 5 & 4 & & & \\
\hline
\end{tabular}

Factor III Apoyo y orientación escolar. La dimensión hace referencia a los comportamientos que muestran el involucramiento de los padres en las responsabilidades escolares y otras actividades de los hijos con el propósito de alentar su un buen desempeño académico.

Factor IV Control conductual. Mide el control y supervisión del comportamiento de los hijos a través de la comunicación y fomento de la confianza. 
Para la subescala de Papá se obtuvo un Índice de adecuación del muestreo Kaiser-Meyer y Olkin KMO de 0.944 y un test de esfericidad de Bartlett de 6415.973; $p=.000$. Se determinó que los siete factores encontrados explicaban
$55.65 \%$ de la varianza total acumulada después de la rotación. En este caso al analizar la matriz de estructura factorial, se observó que los siete factores estaban conformados por al menos tres reactivos (ver tabla 2).

\section{Tabla 2}

Matriz de estructura factorial de la Escala de Percepción de Prácticas de Crianza para Adolescentes para la subescala de Papá

\begin{tabular}{|c|c|c|c|c|c|c|c|}
\hline \multirow[t]{2}{*}{ Reactivo } & \multicolumn{7}{|c|}{ Factores } \\
\hline & 1 & 2 & 3 & 4 & 5 & 6 & 7 \\
\hline 59. Me da palabras de aliento & .738 & .122 & .095 & .049 & -.017 & -.058 & .308 \\
\hline 55. Me muestra cariño (abraza, besa, apapacha) & .719 & .130 & .170 & .118 & -.001 & -.026 & .028 \\
\hline 53. Me muestra comprensión & .717 & .251 & .165 & .148 & .062 & .100 & .051 \\
\hline 51. Me apoya cuando lo necesito & .711 & .155 & .239 & .015 & .027 & .014 & .092 \\
\hline 45. Trata de entender lo que me sucede & .678 & .176 & .134 & .321 & .212 & .051 & -.024 \\
\hline 62. Me hace saber que soy importante para la familia & .676 & .020 & .338 & .069 & .131 & .161 & .131 \\
\hline 34. Me brinda confianza & .664 & .278 & .118 & .111 & .148 & .180 & .214 \\
\hline 40. Me pregunta qué me sucede cuando estoy triste & .656 & .148 & .079 & .226 & .287 & .112 & .054 \\
\hline 43. Me dice lo orgulloso que está de mi & .644 & .073 & .191 & .140 & .113 & .212 & .183 \\
\hline 42. Está enterado cuando tengo problemas & .643 & .139 & .072 & .330 & .117 & .080 & -.060 \\
\hline 18. Me dice que me quiere & .634 & .134 & .121 & .123 & -.007 & .210 & .070 \\
\hline 49. Toma tiempo para platicar conmigo & .632 & .160 & .061 & .306 & .198 & .186 & -.061 \\
\hline 60. Me enseña a cuidar mi salud & .605 & .127 & .359 & .133 & -.011 & -.132 & .183 \\
\hline 6. Me escucha cuando necesito contarle algo & .593 & .358 & -.010 & .202 & .159 & .195 & .085 \\
\hline 36. Me dice que le pregunte si tengo dudas de algo & .575 & .205 & .166 & .172 & .035 & .011 & .241 \\
\hline 23. Me hace sentir que puedo contar con él & .538 & .258 & .208 & .117 & .265 & .243 & -.135 \\
\hline 35. Me inculca valores & .504 & .333 & .425 & .109 & .058 & .145 & .271 \\
\hline 20. Me ayuda a resolver los problemas personales que tengo & .492 & .389 & -.026 & .371 & .210 & .151 & .065 \\
\hline 39. Me explica por qué algo es incorrecto & .489 & .404 & .330 & .202 & .195 & .036 & -.013 \\
\hline 48. Se asegura que vaya diariamente a la escuela & .479 & .055 & .435 & .170 & .133 & -.125 & -.014 \\
\hline 54. Me enseña a esforzarme & .461 & .322 & .366 & .050 & .059 & .075 & .378 \\
\hline 12. Me hace ver las consecuencias de mis actos & .123 & .697 & .100 & .080 & .097 & .086 & .115 \\
\hline 25. Me hace notar mis errores en la convivencia de los demás & .250 & .631 & -.061 & -.074 & .034 & -.138 & -.092 \\
\hline 2. Me hace ver cuáles son mis responsabilidades & .050 & .609 & .269 & .224 & -.042 & .058 & -.014 \\
\hline 32. Me corrige cuando cometo un error & .337 & .538 & .331 & .049 & .130 & -.076 & -.030 \\
\hline 16. Me enseña a respetar a los demás & .278 & .518 & .459 & .054 & .145 & .161 & .177 \\
\hline 38. Me compra los útiles que necesito & .158 & .083 & .713 & .156 & .117 & .137 & .070 \\
\hline 41. Me hace ver la importancia de la escuela & .398 & .139 & .631 & .161 & .082 & .093 & -.166 \\
\hline 37. Me llama la atención cuando desobedezco & .149 & .348 & .501 & .184 & .137 & -.199 & .012 \\
\hline
\end{tabular}


Continuación tabla 2

Reactivo
21. Me dice lo que está bien o lo que está mal
46. Se pone a estudiar conmigo
63. Platica con los maestros sobre cómo voy en la escuela
15. Me ayuda a hacer la tarea
27. Me pone el ejemplo con su propia conducta
22. Me explica las reglas que hay en la casa
29. Me dice que le diga a qué hora regreso en la noche
44. Me pide que le diga a dónde voy cuando salgo
13. Me pide que le diga con quién paso mi tiempo libre
3. Me ignora, no me habla
4. Me dice groserías y malas palabras
57. Me prohíbe hacer ciertas cosas que están mal
8. Me dice que puedo tener amigos en la casa, aun cuando no hay adultos
en ella
10. Me anima a seguir adelante a pesar de todo
5. Me premia cuando hago algo bueno
Porcentaje de Varianza Explicada
Alfa de Cronbach
Media
Desviación estándar
Número de reactivos

\begin{tabular}{lllllll}
\multicolumn{7}{c}{ Factores } \\
$\mathbf{1}$ & $\mathbf{2}$ & $\mathbf{3}$ & $\mathbf{4}$ & $\mathbf{5}$ & $\mathbf{6}$ & $\mathbf{7}$ \\
.355 & .429 & .430 & .157 & .080 & .042 & .126 \\
.349 & -.006 & .114 & .689 & -.033 & .089 & .138 \\
.405 & .045 & .173 & .639 & -.015 & -.080 & -.035 \\
.289 & .071 & .133 & .626 & .049 & .071 & .278 \\
-.013 & .272 & .063 & .456 & .122 & .127 & .238 \\
.224 & .338 & .254 & .400 & .259 & .029 & -.047 \\
.059 & .052 & .223 & .063 & .772 & .012 & .197 \\
.225 & .071 & .408 & -.009 & .618 & -.121 & .088 \\
.258 & .338 & -.165 & .130 & .509 & .006 & -.072 \\
-.351 & -.003 & -.079 & -.038 & -.089 & -.644 & -.030 \\
-.247 & -.100 & -.097 & -.024 & .072 & -.637 & -.043 \\
.227 & .214 & .344 & -.167 & .106 & -.514 & -.034 \\
.057 & -.088 & -.110 & .183 & .042 & .030 & .526 \\
& & & & & & \\
.294 & .308 & .296 & -.071 & .192 & .274 & .436 \\
.376 & .068 & .142 & .150 & .100 & .003 & .416 \\
21.47 & 8.56 & 7,72 & 6.26 & 4.25 & 3.79 & 3.58 \\
0.95 & 0.73 & 0.70 & 0.72 & 0.57 & 0.38 & 0.44 \\
3.97 & 4.01 & 4.47 & 3.02 & 3.54 & 2.31 & 3.21 \\
0.86 & 0.77 & 0.69 & 0.92 & 0.95 & 0.66 & 0.85 \\
21 & 5 & 4 & 5 & 3 & 3 & 3 \\
\hline & & & & & &
\end{tabular}

Los factores obtenidos para la subescala de Papá se definieron de la siguiente manera:

Factor I Cuidado y calidez. Al igual que para Mamá, este factor hace referencia a las conductas que demuestran interés en el bienestar del hijo con muestras de afecto, apoyo y orientación. Factor II Transmisión de valores. Este factor también coincide con el de Mamá y mide las conductas que promueven en los hijos una actitud ética ante la vida y enseñanza de valores. Factor III Disciplina inductiva. En esta dimensión se evalúa el uso de explicaciones acerca de las razones por las cuales los padres consideran que una conducta es deseable o indeseable, con la intención de modificar el comportamiento del hijo.

Factor IV Apoyo y orientación escolar. La dimensión hace referencia a los comportamientos que muestran el interés de los padres en las responsabilidades escolares y otras actividades de los hijos con el propósito de ayudarlos en su desempeño académico. Dicha dimensión también coincide con la subescala de Mamá. Factor V Control conductual. Mide el control y supervisión del comportamiento de los hijos a través de la comunicación y fomento de la con- 
fianza. Este factor también se evalúa en la subescala de Mamá.

Factor VI Retirada de afecto. Evalúa la forma de controlar la conducta basada en conductas como el rechazo, la negación a escucharle, el aislamiento o las amenazas de abandono. Aquí, el poder de la disciplina reside en el miedo a perder el apoyo afectivo, emocional y la aprobación de los padres.

FactorVII Promoción de la autonomía/confianza. Esta última dimensión mide las conductas que promueven la autonomía del hijo en diversos contextos, reforzando sus comportamientos positivos.

Posteriormente, el análisis descriptivo mostró que para el caso de los factores de la subescala de Mamá, las medias más altas se obtuvieron en los factores Transmisión de valores $(M=4.46)$ seguida por el factor Cuidado y calidez $(M=4$. cala de Papá, las medias más altas se obtuvieron en los factores: Disciplina inductiva $(M=4.47)$, Transmisión de valores $(M=4.01)$ y Cuidado y calidez $(M=3.97)$.

Enseguida se procedió a obtener un análisis de diferencias por medio de la prueba $t$ de student para cada uno de los factores de las subescalas de Mamá y Papá según el sexo. No se encontraron diferencias significativas para la subescala de Mamá en ninguno de los factores. En tanto, para la subescala de Papá solo se observaron diferencias significativas en el factor de Apoyo y orientación escolar $\left(t_{(324)}=2.29 ; p=\right.$ $.02)$, donde las adolescentes mujeres $(M=3.17)$ perciben que Papá les apoya en las responsabilidades escolares y otras actividades para que logren un buen desempeño académico a diferencia de los adolescentes varones $(M=2.94$; ver tabla 3).

22). En el caso de las dimensiones de la subes-

Tabla 3

Análisis de varianza obtenido para cada uno de los factores de la Escala de Percepción de Prácticas de Crianza para Adolescentes para la subescala de Mamá y la subescala de Papá por sexo

\begin{tabular}{|c|c|c|c|c|}
\hline \multirow{2}{*}{$\begin{array}{c}\text { Factores } \\
\text { Mamá }\end{array}$} & \multicolumn{2}{|c|}{ Medias } & \multirow[t]{2}{*}{$t$} & \multirow[t]{2}{*}{ Prob. } \\
\hline & Hombres & Mujeres & & \\
\hline Cuidado y calidez & 4.28 & 4.23 & 0.57 & 0.56 \\
\hline Transmisión de valores & 4.43 & 4.54 & 1.82 & 0.06 \\
\hline Apoyo y orientación escolar & 3.30 & 3.26 & 0.46 & 0.64 \\
\hline Control conductual & 3.64 & 3.77 & 1.47 & 0.14 \\
\hline \multicolumn{5}{|l|}{ Factores } \\
\hline \multicolumn{5}{|l|}{ Papá } \\
\hline Cuidado y calidez & 4.10 & 3.93 & 1.72 & 0.08 \\
\hline Transmisión de valores & 4.08 & 4.00 & 0.92 & 0.35 \\
\hline Disciplina inductiva & 4.49 & 4.49 & 0.07 & 0.93 \\
\hline Apoyo y orientación escolar & 3.17 & 2.94 & 2.29 & $0.02^{*}$ \\
\hline Control conductual & 3.50 & 3.61 & 1.09 & 0.27 \\
\hline Retirada del afecto & 2.32 & 2.31 & 0.14 & 0.88 \\
\hline Promoción de la autonomía/confianza & 3.26 & 3.21 & 0.55 & 0.57 \\
\hline
\end{tabular}

${ }^{*} p \leq 0.05{ }^{* *} p \leq 0.01{ }^{* * *} p \leq 0.001$ 
El análisis de varianza de una sola vía fue obtenido por estado para cada uno de los factores de las subescalas de Mamá y Papá, los resultados evidencian que para la subescala de Mamá solo se determinaron diferencias en el factor de Apoyo y orientación escolar $\left(F_{(5,390)}=3.23\right.$; $p=0.007)$, sin embargo, la prueba a posteriori de Scheffé no reveló diferencias significativas entre las medias de los grupos. Por el contrario, en la subescala de Papá se estimaron diferencias en cuatro factores: Cuidado y calidez $\left(F_{(5,314)}=\right.$ 2.97; $p=0.012$ ), en este caso Scheffé mostró que el Estado de México $(M=4.28)$ es el que difiere significativamente del Estado de Sonora $(M=$ $3.57)$; Transmisión de valores $\left(F_{(5,367)}=2.32 ; p\right.$ $=0.04)$ y Disciplina inductiva $\left(F_{(5,373)}=2.62 ; p=\right.$ $0.024)$, en éstos dos factores Scheffé no mostró diferencias significativas entre las medias de los estados. Finalmente, en el factor de Apoyo y orientación escolar $\left(F_{(5,353)}=5.03 ; p=0.000\right)$, donde Scheffé mostró que el Estado de México $(M=3.49)$ es el que difiere significativamente de los estados de Yucatán $(M=2.89)$, Sonora $(M$ $=2.65)$ e Hidalgo $(M=2.86$; ver tabla 4$)$.

Tabla 4

Análisis de varianza obtenido para cada uno de los factores de la Escala de Percepción de Prácticas de Crianza para Adolescentes para la subescala de Mamá y la subescala de Papá por Estado

\begin{tabular}{|c|c|c|c|c|c|c|c|c|}
\hline \multirow{2}{*}{$\begin{array}{l}\text { Factores } \\
\text { Mamá }\end{array}$} & \multicolumn{6}{|c|}{ Medias } & \multirow[t]{2}{*}{$F$} & \multirow[t]{2}{*}{ Prob. } \\
\hline & Yucatán & $\begin{array}{c}\text { Estado de } \\
\text { México }\end{array}$ & Hidalgo & Zacatecas & Coahuila & Sonora & & \\
\hline Cuidado y calidez & 4.29 & 4.42 & 4.29 & 4.10 & 4.14 & 4.04 & 2.04 & 0.07 \\
\hline Transmisión de valores & 4,53 & 4.58 & 4.44 & 4.45 & 4.42 & 4.28 & 1.98 & 0.08 \\
\hline Apoyo y orientación escolar & 3.15 & 3.63 & 3.10 & 3.15 & 3.44 & 3.17 & 3.23 & $0.007^{* *}$ \\
\hline Control conductual & 3.71 & 3.68 & 3.52 & 3.69 & 3.95 & 3.83 & 1.17 & 0.13 \\
\hline \multicolumn{9}{|l|}{$\begin{array}{c}\text { Factores } \\
\text { Papá }\end{array}$} \\
\hline Cuidado y calidez & 3.96 & 4.28 & 3.99 & 4.00 & 3.85 & 3.57 & 2.97 & $0.012^{*}$ \\
\hline Transmisión de valores & 4.11 & 4.20 & 3.94 & 3.99 & 3.95 & 3.68 & 2.32 & $0.04^{*}$ \\
\hline Disciplina inductiva & 4.51 & 4.70 & 4.35 & 4.51 & 4.35 & 4.27 & 2.62 & $0.024^{*}$ \\
\hline Apoyo y orientación escolar & 2.89 & 3.49 & 2.86 & 3.03 & 3.15 & 2.65 & 5.03 & $0.000^{* * *}$ \\
\hline Control conductual & 3.40 & 3.53 & 3.42 & 3.69 & 3.77 & 3.46 & 1.76 & 0.12 \\
\hline Retirada del afecto & 2.33 & 2.14 & 2.40 & 2.24 & 2.50 & 2.29 & 2.00 & 0.07 \\
\hline $\begin{array}{c}\text { Promoción de la autonomía/ } \\
\text { confianza }\end{array}$ & 3.24 & 3.40 & 3.13 & 3.27 & 3.12 & 3.04 & 1.23 & 0.29 \\
\hline
\end{tabular}

${ }^{*} p \leq 0.05^{* *} p \leq 0.01{ }^{* * *} p \leq 0.001$ 


\section{Discusión}

Los resultados obtenidos en la validación de la escala mostraron que los factores conformados -aunque diferenciados para Papá y Mamá-, reflejan las dimensiones básicas de la crianza propuestas por la literatura: el control y el afecto o calidez. No obstante, es de notar que para Mamá se obtienen cuatro factores, en tanto que para Papá se establecen siete. Los cuatro para la subescala de Mamá fueron determinados a su vez para la subescala de Papá. Para este último los factores de disciplina inductiva, retirada del afecto (como otra forma de control) y la promoción de la autonomía. Este resultado sugiere que los papás son percibidos con un papel más activo en el ejercicio del control y monitoreo de sus hijos adolescentes en comparación con las Mamás.

Los análisis descriptivos, a su vez, evidencian una percepción de la Mamá y del Papá como figuras afectuosas, que imponen límites, inculcan valores y autocontrol, además de proveer calidez, lo cual correspondería al estilo autoritativo de crianza. Asimismo, no se observan diferencias entre los y las adolescentes respecto a la percepción del Papá y Mamá, con excepción del factor apoyo y orientación escolar, donde los adolescentes perciben que su Papá les apoya en lo relativo a la escuela más que a las adolescentes. Hombres y mujeres adolescentes perciben la retirada del afecto como una estrategia poco usada por sus Papás.

Por último, en cuanto a las diferencias por zona geográfica, se obtuvo que los y las ado- lescentes del Estado de México perciben más apoyo y calidez del papá que los de Sonora (quienes obtienen las puntuaciones más bajas en este factor), y más apoyo y orientación escolar que los de Yucatán, Hidalgo y Sonora.

Estos resultados evidencian que durante la adolescencia, los padres contribuyen al desarrollo psicosocial de sus hijos, al relacionarse con ellos a través del uso de diversas estrategias de socialización -más o menos coercitivas, con mayor o menor demostración de afecto-, que son percibidas por los hijos, y conllevan importantes consecuencias en su ajuste psicológico (Bosco et al., 2003; Forehand \& Nousiainen, 1993; Méndez, Andrade, \& Peñaloza, 2013; Roa \& Del Barrio, 2002).

Merecen ser resaltadas las diferencias encontradas en la percepción de Padres y Madres de los diferentes contextos, en consonancia con la literatura, según la cual los factores culturales, sociales y familiares moldean las prácticas específicas que se ejercen y que son percibidas por los hijos (Ramírez, 2009; Rodrigo \& Palacios, 1998). Por otra parte, se obtiene que las Madres son percibidas, en general, como más cálidas y más controladoras que los Padres, tal resultado es consistente con otros estudios (Rodríguez, Del Barrio, \& Carrasco, 2009; Paulson, Hill, \& Holmbeck, 1991; Paulson \& Sputa, 1996).

Los resultados obtenidos en este estudio ofrecen, por una parte, un instrumento cultualmente válido y confiable, para la percepción de las prácticas de crianza paternas, y por otra parte, otorgan información sobre este 
importante constructo en la muestra estudiada. Empero, entre las limitaciones de este estudio se encuentran los resultados obtenidos para la subescala de Papá, ya que los últimos dos factores obtienen consistencias internas muy bajas, por lo cual se hace necesario verificar dichos factores en el futuro. Otras limitaciones es el número de estados que participaron en la muestra, seis, además el rango de edad de los sujetos es limitado (adolescentes de secundaria), en consecuencia, los resultados no son válidos para otros adolescentes (de preparatoria). Estas limitaciones deberán considerarse para estudios posteriores, aunque la medición del constructo en estas regiones del país con dicho instrumento constituye un primer paso para definir conceptual y empíricamente el constructo; aún queda mucho trabajo que realizar para su medición.

\section{Referencias}

Aguilar, J. (2003). Influencia de los estilos parentales sobre el desarrollo psico-social de niños y jóvenes. Ponencia presentada en el $I X$ Congreso Mexicano de Psicología. México: Sociedad Mexicana de Psicología.

Aguilar, V. J., Valencia, C. A., \& Romero, S. P. (2004). Estilos parentales y desarrollo psicosocial en estudiantes de bachillerato. Revista Mexicana de Psicología, 21, 119-129. Aguirre, E. (2000). Socialización y prácticas de crianza. En E. Aguirre \& E. Durán, (Eds.) Socialización: Prácticas de crianza y cuidado de la salud. Bogotá: CES-Universidad Nacional de Colombia.

Alonso, G. J., \& Román, S. J. Ma . (2005). Prácticas educativas familiares y autoestima. Psicothema, 17(1), 76-82.

Baumrind, D. (1971). Current patterns of parental authority. Developmental Psychology Monograph, 4, 1-103.

Baumrind, D. (1975). The contribution of the family to the development of competence in children. Schizophrenia Bulleltin, 14, 12-37.

Baumrind, D. (1980). New directions in socializiation research. American Psychologist, 35, 639-650.

Baumrind, D. (1991). Parenting styles and adolescent development. In J. Brooks-Gunn, R. Lenner \& A. C. Peterson (Eds.), The Encyclopedia of Adolescent (pp. 746-758). New York: Garland.

Balzano, S. (2002). Las construcciones culturales sobre el éxito y el fracaso escolar y sus implicaciones sobre los modelos educativos en Argentina. Cultura y Educación, 3(14), 283-296.

Becker, W. C. (1964). Consequences of different kinds of parental discipline. In $\mathrm{M}$. L. Hoffman \& L.W. Hoffman (Eds.), Review of child development research (Vol. 1. Cap. 9). New York: Russell Sage Foundation.

Betancourt, O. D., \& Andrade P. P. (2011). Control parental y problemas emocionales y de conducta en adolescentes. Revista Colombiana de Psicología, 20(1), 27-41. 
Block, J. H. (1981). The Child Rearing Practices Report: A set of items for the description of parental socialization attitudes and values. Berkeley: Institute of human development, University of California.

Bornstein, M. H., \& Sawyer, J. (2005). Family systems. In K. McCartney \& D. Phillips (Eds.), Blackwell handbook on early childhood development (pp. 381-398). Malden, MA: Blackwell.

Bosco, G. L., Renk, K., Dinger, T. M., Epstein, K. M., \& Phares, V. (2003). The connections between adolescents' perceptions, parental psychological symptoms, and adolescent functioning. Applied Developmental Psychology, 24, 179-200.

Bronfenbrenner, U. (1979). The ecology of Human Development. Cambridge: Harvard University Press.

Buri, J. R. (1991). Parental Authority Questionnaire. Journal of Personality Assessment, 57, 110-119.

Carrasco, M. A., Holgado, F. P., Del Barrio, M. V., \& Barbero, I. (2007). Validez incremental: Un estudio aplicado con diversas fuentes informantes y medidas. Acción Psicológica, 5(2), 67-76.

Carrasco, M. A., Holgado, P., \& Del Barrio, V. (2007). ¿Cómo perciben los hijos la crianza materna y paterna? Escritos de Psicología, 2(2), 10-18.

Cava, Ma J., Musitu, G., \& Murgui, S. (2006). Familia y violencia escolar: El rol mediador de la autoestima y la actitud hacia la auto- ridad institucional. Psicothema, 18(3), 367373.

Ceballos, E. M., \& Rodrigo, M. J. (1998). Las metas y estrategias de socialización entre padres e hijos. En M. J. Rodrigo \& J. Palacios (Coords.), Familia y desarrollo humano (pp. 225-242). Madrid: Alianza Editorial.

Craig, G. (2008). Desarrollo Psicológico. México: Prentice Hall.

Darling, N., \& Steinberg, L. (1993). Parenting style as a context: An Integrative Model. Psychologycal Bulletin, 113, 487-496.

Eraso, J., Bravo, Y., \& Delgado, M. (2006). Creencias, actitudes y prácticas sobre crianza en madres cabeza de familia en Popayán: Un estudio cualitativo. Revista de Pediatría, 41(3), 23-40.

Finley, G. E., \& Schwartz, S. J. (2004). The Father Involvement and Nurturant Fathering Scales: Retrospective measures for adolescent and adult children. Educational and Psychological Measurement, 64, 143-164.

Flores, G. M., Cortés, A. L., \& Góngora, C. E. (2008). Familia, crianza y personalidad. Yucatán: UADY-Facultad de Psicología.

Forehand, R., \& Nousiainen, S. (1993). Maternal and paternal parenting: Critical dimensions in adolescent functioning. Journal of Family Psychology, 7, 213-221.

Frías, A. M., Sotomayor, P. M., Corral, V. V., \& Castell, R. I. (2004). Parental Styles and Harsh Parenting in a Sample of Mexican Women: A Structural Model. Interamerican Journal of Psychology, 38(1), 61-72. 
Gaylord, N. K., Kitzmann, M., \& Coleman, J. K. (2003). Parent and children's perceptions of parental behaviour: Associations with children's psychological adjustment in the classroom. Parenting: Science and Practice, 3(1), 23-47.

Goodnow, J. J., \& Collins, W. A. (1990). Development according to parents: The nature, sources, and consequences of parent'ideas. Hillsdale, NJ: Erlbaum.

González, N., Cauce, A. M., \& Mason, C. (1997). Interobserver agreement in the assessment of parental behavior and parent-child conflict: African American mothers, daughters, and independent observes. Child Development, 67, 1483-1498.

Grusec, J., \& Goodnow, J. (1994). Impact of parental discipline methods on the child's internalization of values: A reconceptualization of current points of view. Developmental Psychology, 30(1), 4-19.

Ivanova, M., \& Israel, A. (2006). Family stability as a protective factor against psychopathology for urban children receiving psychological services. Journal of Clinical Child and Adolescent Psychology, 35, 564-570.

Jiménez, M., Hernández-Guzmán, L., \& Reidl, M. (2001). Prácticas de crianza materna percibidas por niños pequeños. Revista Mexicana de Psicología, 18(2), 257-266.

Kerlinger, F., \& Lee, H. (2002). Investigación del comportamiento. México: McGraw Hill/ Interamericana.
Maccoby, E. E. (1984). Middle childhood in the context of the family. In W. A. Collins (Ed.), Development during middle childhood. Washington, DC: National Academy Press.

Maccoby, E. E., \& Martin, J. A. (1983). Socialization in the context of the family: parentchild interactions. In $\mathrm{E}$. M. Hetherington \& P. H. Mussen (Eds.), Socialization, personality and social development. Handbook of child psychology (Vol. IV. Cap. 9. pp. 1-102). New York: Wiley. McKinney, C., Milone, M. C., \& Renk, K. (2011). Parenting and late adolescent emotional adjustment: Mediating effects of discipline and gender. Child Psychiatry Human Development, 42(4), 463-481. doi: 10.1007/ s10578-011-0229-2

Méndez, S. M., Andrade, P. P., \& Peñaloza, R. (2013). Prácticas parentales y capacidades y dificultades en preadolescentes. Revista intercontinental de Psicología y Educación, 15(1), 99-118.

Mestre, M., Pérez-Delgado, E., Tur, A., Diez, I., Soler, J., \& Samper, P. (1999). El razonamiento prosocial en la infancia y en la adolescencia. Un estudio empírico. En E. PérezDelgado \& M. Mestre (Eds.), Psicología moral y crecimiento personal. España: Ariel. Michaels, G. Y., Meese, L. A., \& Stollak, G. E. (1983). Seeing parental behavior through different eyes: Exploring the importance of person perception processes in parents and children. Genetic Psychology Monographs, 107, 36-60. 
Molpereces, P. Ma A., Llinares, I. L. I., \& Musitu, O. G. (2001). Estilos de disciplina familiar y prioridades de valor en la adolescencia. $R e$ vista de Psicología Social Aplicada, 11(3), 49-67.

Montiel, M., Vera, J. A., Peña, M., Rodríguez, A., \& Félix, J. (2002). Estrés en la crianza, número de hijos y edad de la madre. La Psicología Social en México, 9, 856-861.

Morris, A., Silk, J., Steinberg, L., Sessa, F., Avenevoli, S., \& Essex, M. (2002). Temperamental vulnerability and negative parenting as interacting predictors of child adjustment. Journal of Marriage and family, 64(2), 461471.

Musitu, G., \& Soledad-Lila, M. (1993). Estilos de socialización e intensidad de la comunicación en las relaciones padres-hijos. Revista de Psicología Universitas Tarragonensis, 15(2), 5-29.

Oliva, A., Parra, A., \& Arranz, E. (2008). Estilos relacionales parentales y ajuste del adolescente. Infancia y Aprendizaje, 31(1), 93-106.

Oudhof, H., González, N., Rodríguez, B., \& Unikel, C. (2008). Escala de Percepción de Crianza para Jóvenes. Toluca: Universidad Autónoma del Estado de México.

Palacios, D. J. R., \& Andrade P. P. (2006). Escala de estilos parentales en adolescentes mexicanos. Revista de Psicología Social y Personalidad, 22, 49-64.

Palacios, J., \& Moreno, M. (1999). Contexto familiar y desarrollo social. En J. Rodrigo
(Ed.), Contexto y desarrollo social (pp. 157188). Madrid: Síntesis.

Palacios, J., González, M. M., \& Moreno, M. C. (1992). Stimulating the child in the zone of proximal development: The role parents' ideas. In I. Sigel, J. Goodnow \& A. V. McGilliculddy-De Lise (Eds.), Parent beliefs systems. The psychological consequences for children (pp. 71-94). Hillsdale, New Jersey: Erlbaum.

Paulson, S. E. (1994). Relations of parenting style and parental involvement with ninthgrade students' achievement. Journal of Early Adolescence, 14, 250-267.

Paulson, E., Hill, J., \& Holmbeck, G. (1991). Distinguishing between perceived closeness and parental warmth in families with seventh-grade boys and girls. Journal of Early Adolescence, 11(2), 276-293.

Paulson, E., \& Sputa, L. (1996) Patterns of parenting during adolescence: Perception of adolescents and parents. Adolescence, 31, 369-381.

Peralbo. U. M., \& Fernández, A. Ma. L. (2003). Estructura familiar y rendimiento escolar en Educación Secundaria Obligatoria. Revista Galego Portuguesa de Psicoloxía e Educación, 7(8), 309-322.

Pérez, R. M., \& Aguilar, V. J. (2009). Relaciones del conflicto padres-adolescentes con la flexibilidad familiar, comunicación y satisfacción marital. Psicología y Salud, 19(1),111-120.

Pérez, R. M., \& Alvarado, M. C. (2015). Los Estilos Parentales: Su Relación en la Nego- 
ciación y el Conflicto entre Padres y Adolescentes. Acta de Investigación Psicológica- Psychological Research Records, 5(2), 1972-1983.

Perron, R., \& Mathon, T. H. (1976). Love and authority perceptions of parental figures in verbal accounts of children. Psychology Francaise, 21(3), 131-157.

Pipp, S., Shaver, P., Jennings, S., Lamborn, S., \& Fisher K. W. (1985). Adolescents' theories about the development of their relationships with parents. Journal of personality and social psychology, 48(4), 991-1001.

Ramírez, C. M. A. (2009). Familia, escenario de socialización. International Journal of developmental and Educational Psychology, 1(2), 427-434.

Rivera, H. M. E., \& Andrade, P. P. (1998). Las dimensiones de la familia en México. La Psicología Social en México, 7, 222-227.

Rivera, H. M. E., \& Andrade, P. P. (2010). Escala de Evaluación de las Relaciones Intrafamiliares (ERI). Uaricha Revista de Psicología, 14, 12-29.

Roa, L., \& Del Barrio, V. (2002). Cuestionario de percepción de la crianza para niños y adolescentes. Psicología Educativa, 8, 37-51.

Rodrigo, M. A. J., \& Palacios, J. (Coords.). (1998). Familia y desarrollo humano. Madrid: Alianza Editorial.

Rodrigo, M. A. J., García, M., Márquez, M. L., \& Triana, B. (2005). Discrepancias entre padres e hijos adolescentes en la frecuencia percibida e intensidad emocional en los conflictos familiares. Estudios de Psicología, 26(1), 21-34.

Rodríguez, M. A. J., Del Barrio, M. V., \& Carrasco, M. A. (2009). ¿Cómo perciben los hijos la crianza materna y paterna? Diferencias por edad y sexo. Escritos de Psicología, 2(2) 10-18.

Rodríguez, A. B., Oudhof van Barneveld, H., González-Arratia, I., \& Unikel, S. C. (2011). Desarrollo de una escala para medir la percepción de crianza parental en jóvenes estudiantes mexicanos. Pensamiento Psicológico, 9(17), 9-20.

Rohner, R. P., \& Veneziano, R. A. (2001). The importance of father love: History and contemporary evidence. Review of General Psychology, 5, 382-405.

Samper, P., Cortés, M. T., Mestre, M. V., Nácher, M. J., \& Tur, A. (2006). Adaptación en población española del Child's Report of Parent Behavior Inventory. Psicothema 18(2), 263-271.

Schaefer, E. S. (1965). Children's reports of parental behavior: An inventory. Child Development, 36(2), 413-424.

Shek, D. (1998). Perceptions of parenting styles and parent-adolescent conflict in adolescents with low academic achievement in Hong Kong. Social Behavior and Personality, 26, 89-98.

Shek, D. (2000). Differences between fathers and mothers in the treatment of, and relation- 
ship with, their teenage children: Perceptions of Chinese adolescents. Adolescence, 35, 135-146.

Sorbring, E., Rödholm-Funnemark, M., \& Palmerus, K. (2003). Boys' and gilrs' perceptions of parental discipline in transgression situations. Infant and Child Development, 12, 53-69.

Spera, C. (2005). A review of the relationship among parenting practices, parenting styles, and adolescents school achievement. Educational Psychology Review, 17(2), 125-146.

Steinberg, L. (2001). We know these things: Parent adolescent relationships in retrospect and prospect. Journal of Research on Adolescence, 11, 11-19.

Touliatos, J., Perlmutter, B. F., \& Straus, M. A. (2001). Handbook of family measurement techniques. Thousand Oaks, CA: Sage Publications, Inc.

Vallejo, C. A. (2002). Estilos parentales y conflictos de autoridad entre padres y adolescentes totonacas en el medio rural (Tesis inédita de Doctorado en Psicología). Universidad Nacional Autónoma de México, México.

Villegas-Pantoja, M. A., Alonso-Castillo, M. M., Alonso-Castillo, B. A., \& Martínez-Maldonado, R. (2014). Percepción de crianza parental y su relación con el inicio del consumo de drogas en adolescentes mexicanos. Aquichan, 14(1), 41-52.
Recibido el 08 de septiembre de 2015

Revisado el 08 de enero de 2016

Aceptado el 02 de marzo de 2016 